\title{
An introduction to the statistical physics of active matter: motility-induced phase separation and the "generic instability" of active gels
}

\author{
Davide Marenduzzo ${ }^{\mathrm{a}}$ \\ School of Physics and Astronomy, University of Edinburgh, Peter Guthrie Tait Road, \\ Edinburgh EH9 3FD, UK
}

Received 10 March 2016 / Received in final form 26 April 2016

Published online 10 November 2016

\begin{abstract}
In this work we review some statistical physics techniques to coarse grain active matter systems, writing down a set of continuum fields which track the evolution of macroscopic fields such as density, momentum, etc. While the method can be applied in general, we will focus here on two simple and by now well-studied, active matter examples. First, we will consider motility-induced phase separation, the phenomenon by which a concentrated suspension of self-propelled particles spontaneously separates into a dense and a dilute phase. Second, we will review the so-called "generic instability" of active gels, which refers to the nonequilibrium phase transition between a quiescent and a spontaneously flowing phase in a concentrated suspension of rodlike active particles. For both these cases, we also outline recent developments in the literature.
\end{abstract}

\section{Introduction: an outline, and a definition of active systems}

In this work we provide an introductory description of the physics of active matter, focussing on some methods which are often used in the field and on statistical physics aspects, mainly nonequilibrium phase transitions. As an underlying example we will mainly consider a collection of self-motile particles, such as swimming bacteria or synthetic microswimmers. These notes are an adaptation of those in Ref. [1].

We will first see how to go from a Boltzmann-like kinetic theory of self-propelled particles to a set of macroscopic (also called hydrodynamic) equations of motion for a set of fields, such as the swimmer density. We will do so by studying the example of a collection of self-propelled particles whose velocity decreases with density: the resulting equations of motion can provide a framework within which to study motilityinduced phase separation, a phase transition which is currently a topical area of research in the field of active matter physics.

We will then discuss how to extend such theories to incorporate solvent-mediated interactions, which are usually important as most self-propelled particles move within

\footnotetext{
${ }^{a}$ e-mail: dmarendu@ph.ed.ac.uk
} 


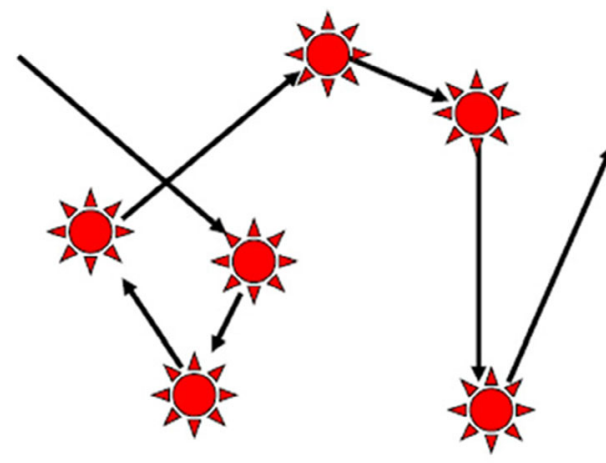

Run:

Tumble:

Fig. 1. Sketch of the run and tumble dynamics typical of bacteria. Figure taken from Ref. [4], with permission. (This figure is subject to copyright protection and is not covered by a Creative Commons license.)

a fluid. These hydrodynamic interactions can be incorporated either by large scale computer simulations of fully-resolved or point-like swimmers, or by writing down macroscopic equations based on the coarse graining of a more microscopic theory. We will see that there is a generic instability which is well described by these equations, between a quiescent suspension and one which spontaneously flows, and we will describe how this solvent-mediated phase transition is currently understood.

Before proceeding, we need some basic definitions. For our purposes, active matter will be a collection of active particles; these are "particles which absorb energy from their surroundings or from an internal fuel tank and dissipate it to engage in a variety of non-equilibrium activities, usually, but not solely, connected to motility, growth or replication" (this definition is an adaptation from those adopted in Refs. [2,3]).

According to these definitions, examples of active matter as collections of active particles are: suspensions of swimming bacteria, or of other motile microorganisms, cell suspensions, and collections of cytoskeletal filaments, such as filamentous actin or microtubules, and molecular motors, such as myosin or kinesin. Activity is often (but not solely) linked to self-motility: it could also come, for instance, from selfreplication, in bacterial or microbial systems, or from polymerisation and from nonthermal motor-exerted forces, in the context of actin and myosin, or in general in cytoskeletal systems.

\section{A simple example: effective diffusion in run-and-tumble bacteria}

Let us begin by considering a solution of swimming bacteria (E. Coli for concreteness). The way bacteria such as $E$. coli move is via a characteristic run-and-tumble dynamics (Fig. 1). Essentially, these bacteria move in a straight line during a "run", at a velocity $v \sim 10-30 \mu \mathrm{m} / \mathrm{s}$, then change direction (to a first approximation randomly) in a "tumble", after which they move straight for another "run", etc. The duration of each run, $\tau$ (which also equals the time lapse between two successive tumbles) is on average about $1 \mathrm{~s}$, while the "tumble" is much faster (about an order of magnitude), hence it can be modelled, to begin with, as instantaneous. 
It is intuitively apparent that, after many tumbles, the motion of a single swimming bacterium is effectively diffusive, and the diffusion coefficient can be estimated quite simply by using the standard formula linking the mean square displacement of a particle in 3D (here the run-and-tumble bacterium), $\left\langle\mathbf{r}^{2}\right\rangle$, and time, $t$

$$
6 D_{\text {eff }} t=\left\langle\mathbf{r}^{2}\right\rangle
$$

Because $\mathbf{r}$ is the distance covered after $N$ runs, if we call $\Delta \mathbf{x}_{i}$ the distance covered in the $i$-th run, we also have that

$$
\mathbf{r}=\sum_{i=1}^{N} \Delta \mathbf{x}_{i}
$$

Assuming that the run length, $l_{\text {run }}=v \tau$, is constant, and that the directions of the runs are completely uncorrelated with each other, we obtain

$$
6 D_{\text {eff }}=\sum_{i, j=1}^{N}\left\langle\Delta \mathbf{x}_{i} \cdot \Delta \mathbf{x}_{j}\right\rangle /(N \tau)=\sum_{i, j=1}^{N} \delta_{i j} l_{\text {run }}^{2} /(N \tau)=l_{\text {run }}^{2} / \tau,
$$

where $\delta_{i j}$ denotes the Kronecker delta. We should note here that a better approximation to the run-and-tumble dynamics would be to say that there is a constant probability to tumble. In this case the run length would not be constant, but would become a stochastic variable. This Poissonian run-and-tumble assumption leads to $D_{\text {eff }}=v^{2} \tau / 3$ instead of $D_{\text {eff }}=v^{2} \tau / 6$, so the functional form is the same but the prefactor changes.

Now, imagine that we switch off activity in our run-and-tumble particles, for instance by considering a bath of non-motile bacteria. What would the behaviour of the system be? To answer, we should realise that in the absence of motility the particles would be indistinguishable from colloidal particles of the same size (and shape). Of course also these particles diffuse, and if we approximate them as spheres of diameter $\sigma$, we know that their diffusion coefficient is given by the Stokes-Einstein formula [5],

$$
D=\frac{k_{B} T}{3 \pi \eta \sigma} \sim \frac{4 \times 10^{-21} \mathrm{~J}}{10 \times 10^{-3} \mathrm{Pas} 10^{-6} \mathrm{~m}},
$$

where $\eta$ is the fluid viscosity (here estimated for water) and $\sigma$ is the particle size (here $1 \mu \mathrm{m}$ ). This is much smaller than the effective diffusion of bacteria, $D_{\text {eff }}$, which can be estimated to be 10-100 times larger [1]. This discrepancy means that the Stokes-Einstein formula does not hold for bacteria. The deep reason behind this is that this formula depends crucially on the fluctuation-dissipation theorem, which in turn only holds for systems which are near thermodynamic equilibrium. While this assumption is true of inanimate colloidal particles, it clearly does not hold for swimmers, which use energy from the environment in order to do active work. This simple example already shows that the non-equilibrium nature of active matter renders it fundamentally different from conventional passive soft matter. Here, all this amounts to is a quantitative difference in the effective diffusivity of active particles. In what follows we will see that activity can lead to much more profound consequences and qualitatively different physics.

\section{From Boltzmann-like equations to continuum models: the physics of motility-induced phase separation as an example}

We now consider again our example of a population of run-and-tumble swimmers (Fig. 1), that runs for a time $\tau$ with velocity $v$ between two successive tumbles. Let 
$f(\mathbf{x}, \mathbf{u}, t)$ be the distribution of active particles with position $\mathbf{x}$, swimming in the direction $\mathbf{u}$ at the time $t$. The kinetic (Boltzmann-like) equation for the evolution of $f$ in $d$ dimensions can be written as

$$
\partial_{t} f(\mathbf{x}, \mathbf{u}, t)=-\nabla \cdot[v \mathbf{u} f(\mathbf{x}, \mathbf{u}, t)]-\frac{f(\mathbf{x}, \mathbf{u}, t)}{\tau}+\frac{\int f(\mathbf{x}, \mathbf{u}, t) \mathrm{d}^{d-1} \mathbf{u}}{\tau \underbrace{\int \mathrm{d}^{d-1} \mathbf{u}}_{\text {solid angle } \Omega_{d}}} .
$$

The first term on the right hand side of the equation is a convective term, where $v \mathbf{u}$ is the velocity at $\mathbf{x}$ (note $\nabla$ is the gradient with respect to $\mathbf{x}$ ); the second term is a linear term accounting for the particle that "tumble out" of $\mathbf{u}$, with a rate $\tau$; the same rate appears in the third term, that accounts for the particles "tumbling into" u. (In other words, the second and third term can be viewed as loss and gain terms if we view Eq. (5) as a master equation.) The integral of $f$ appearing in the third term is the zero-th order moment of $f$ (with respect to the orientation $\mathbf{u})$ : this is the local active particle density, $\rho(\mathbf{x}) .\left(\Omega_{d}\right.$ denotes the $d$-dimensional solid angle.)

Equations analogous to Eq. (5) (i.e. Boltzmann-like kinetic equations) are often the starting point of theories of nonequilibrium statistical physics, because they can be written down on the basis of a set of microscopic rules defining a model; they are therefore used many times in active matter physics as well. However, Eq. (5) is rather difficult to deal with analytically. A way to simplify it is to consider the so-called hydrodynamic limit, in which suitable moments of the distribution functions, which are linked to macroscopic observables, change smoothly in space and in time. The idea is that, under these conditions, it is physically meaningful to perform a gradient expansion (which is very similar to the so-called Chapman-Enskog expansion [6]) of Eq. (5), which assumes that successive derivatives (gradients) are smaller and smaller on macroscopic (coarse grained scales). The distribution functions are then written as

$$
f=f^{(0)}+f^{(1)}+f^{(2)}+\ldots
$$

where $f^{n}$ is a contribution which is $\sim \mathcal{O}\left(D^{n}\right)$, where $D$ denotes derivatives over space or time. As anticipated, in the hydrodynamic limit we also assume that every successive derivative applied to $f$ makes it much smaller, so:

$$
\mathcal{O}\left(D^{n}\right) \ll \mathcal{O}\left(D^{n-1}\right) \ll \ldots \ll \mathcal{O}\left(D^{1}\right) \ll \mathcal{O}\left(D^{0}\right) .
$$

Therefore,

$$
f^{(n)} \ll f^{(n-1)} \ll . . \ll f^{(1)} \ll f^{(0)} .
$$

In this way, we can set up a hierarchy of equations at various orders in $D$, by equating terms of order $D^{n}$ in the left and right hand side of Eq. (5). For the zero-th and first order, this leads to the following equalities:

$$
\begin{gathered}
\mathcal{O}\left(D^{0}\right): \quad f^{(0)}=\frac{\rho}{\Omega_{d}} \\
\mathcal{O}\left(D^{1}\right): \quad \partial_{t} f^{(0)}+\nabla \cdot\left(v \mathbf{u} f^{(0)}\right)=-\frac{f^{(1)}}{\tau} .
\end{gathered}
$$

From Eq. (8) we find that no term other than $f^{(0)}$ contributes to the coarse-grained hydrodynamic density $\rho$; from Eq. (9) we get the following expression for $f^{(1)}$ :

$$
f^{(1)}=-\tau\left[\partial_{t} f^{(0)}+\partial_{\beta}\left(v \mathbf{u}_{\beta} f^{(0)}\right)\right] .
$$


Note that here and in what follows Greek indices denote Cartesian coordinates (and we imply summation over repeated indices).

We now want to use this hierarchy of approximations for the distribution functions to get macroscopic equations for the hydrodynamic moments - these are linked to macroscopic observables as we have just seen. The moments we are interested in are the following,

$$
\rho(\mathbf{x}, t)=\int f(\mathbf{x}, \mathbf{u}, t) \mathrm{d}^{d-1} \mathbf{u}
$$

and

$$
\mathbf{j}(\mathbf{x}, t)=\int f(\mathbf{x}, \mathbf{u}, t) v \mathbf{u} \mathrm{d}^{d-1} \mathbf{u} .
$$

Here $\mathbf{j}$ is the density current of swimmers. Now by plugging in the estimates for $f$ (at a certain order in gradient), we can get estimates for these moments. In particular the current $\mathbf{j}$ is estimated as:

$$
\mathbf{j}(\mathbf{x}, t)=\int\left[f^{(0)}(\mathbf{x}, \mathbf{u}, t)+f^{(1)}(\mathbf{x}, \mathbf{u}, t)+\ldots\right] v \mathbf{u d}^{d-1} \mathbf{u} \equiv \mathbf{j}^{(0)}+\mathbf{j}^{(1)}+\ldots
$$

In the simplest case we consider here, it is sufficient to truncate the expansion after $f^{(1)}$, and ignore higher order terms (unfortunately this is not always possible!). From Eq. (8) we get that the zero-th order of the current is zero,

$$
\mathbf{j}^{(0)}=\int \frac{\rho}{\Omega_{d}} v \mathbf{u} \mathrm{d}^{d-1} \mathbf{u}=0,
$$

as it is an integral over $\mathbf{u}$ of an odd function in $\mathbf{u}$. The first order instead, from Eq. (10), is:

$$
j_{\alpha}^{(1)}=-\tau v \int u_{\alpha} \partial_{\beta}\left(v u_{\beta} f^{(0)}\right) \mathrm{d}^{d-1} \mathbf{u}=-\tau v \partial_{\beta}(\rho v) \frac{\int u_{\alpha} u_{\beta} \mathrm{d}^{d-1} \mathbf{u}}{\Omega_{d}} .
$$

It is a small exercise in multidimensional integration using generalised spherical coordinates to show that

$$
\frac{\int u_{\alpha} u_{\beta} \mathrm{d}^{d-1} \mathbf{u}}{\Omega_{d}}=\frac{\delta_{\alpha \beta}}{d}
$$

with $\delta_{\alpha \beta}$ the Kronecker delta.

The resulting hydrodynamic equation for the particle density can be written down by integrating Eq. (5), which is simplified by noting that the integral of the right-hand side vanishes (this is physically due to the fact that the gain and loss terms do not create or destroy particles, so conserve density). By keeping terms up to order $f^{(1)}$ (equivalently $\mathbf{j}^{(1)}$ ), we arrive at an equation which is second order in gradients, and can be worked out to be:

$$
\partial_{t} \rho=\frac{v^{2} \tau}{d} \nabla^{2} \rho=D_{\text {eff }} \nabla^{2} \rho .
$$

Pleasingly, this is the same equation we argued should hold in the introduction, apart from a factor of 2 in the effective diffusivity (which as we anticipated is linked to the fact that here the tumbling rate, rather than interval between tumbles, is constant).

So far, no surprises here. Now consider a simple variation. Instead of considering a constant swimming velocity $v$ over the run, as done in the derivation above, imagine that the velocity depends on position, and it is given by some function $v(\mathbf{x})$. Following the same step in the derivation above, the only important thing to take care of now 
is where exactly the swimming speed ends up in the final equation. We obtain the following hydrodynamic equation for the density $\rho(\mathbf{x})$,

$$
\partial_{t} \rho=\frac{\tau}{d} \nabla \cdot[v \nabla(\rho v)] .
$$

Equation (18) has some important consequences. Most notably, the steady state distribution (obtained by setting $\partial_{t} \rho=0$ ), is $\rho=1 / v(\mathbf{x})$. Therefore run-and-tumble bacteria accumulate where they go more slowly. While this may seem intuitive, no such property holds in conventional passive suspensions of Brownian colloidal particles. Indeed, the analogous case there would be to have a spatially dependent diffusion coefficient, $D(\mathbf{x})$. However, Brownian particles with position-dependent diffusion would not accumulate where they diffuse more slowly; instead their equilibrium distribution will be uniform, because the probability distribution of a Brownian particle in equilibrium (hence its density) is proportional to its Boltzmann weight, $\exp \left(-V / k_{B} T\right)$, where $T$ is temperature, $k_{B}$ is the Boltzmann constant, and $V$ is the potential. When particles are mutually non-interacting (as our run-and-tumble swimmers in Eq. (5)), $V=0$ hence it follows that the density has to be uniform.

This qualitative difference can lead to dramatic differences in the emergent largescale behaviour of run-and-tumble and Brownian particles, and it is at the basis of an interesting phenomenon, known as motility-induced phase separation (also sometimes referred to as self-trapping). To introduce this phenomenon, let us consider the case of a concentrated suspension of run-and-tumble particles, where the swim speed decreases with density, for example, but not necessarily, due to crowding (another mechanism could be quorum-sensing in bacterial systems). To fix the ideas, we consider an exponential decay of the density-dependent swim speed, $v(\rho) \sim \mathrm{e}^{-\lambda \rho / 2} v_{0}$, with $v_{0}$ the speed of a single bacteria (at infinite dilution). Working in $1 \mathrm{~d}$ for simplicity, it is straightforward to see that Eq. (18) then becomes

$$
\partial_{t} \rho=\partial_{x}\left[\tau v^{2}\left(1-\frac{\lambda \rho}{2}\right) \partial_{x} \rho\right]=\partial_{x}\left[D_{\mathrm{eff}}(\rho) \partial_{x} \rho\right]
$$

The effective diffusion coefficient $D_{\text {eff }}$ is now a function of the density. More importantly, for $\rho$ high enough $D_{\text {eff }}<0$, so the effective diffusivity becomes negative. Negative diffusion means that an infinitesimally small fluctuation leads to a divergent increase in the density field. In other words, the system phase separates: this is motility-induced phase separation. (A regularised version of Eq. (19) can be written down by introducing a fourth-order derivative in the right-hand side of Eq. (19), such as, e.g., $-k \partial_{x}^{4} \rho$; this extra term can be motivated as a surface tension between particle-rich and particle-poor domains [7].)

Physically, the motility-induced phase separation described by Eq. (19) can be understood as due to a positive feedback mechanism (this is the self-trapping). Imagine that, due to some small density fluctuation, there is a region where swimmer are more concentrated. In this region, their swim speed will decrease (due to our choice of $v(\rho))$. Now, due to the fact that the swimmers accumulate where they go slower, the local density will increase yet more, leading to a further decrease in velocity and eventually to phase separation. In practice, phase separation only happens for a sufficiently steep decrease of swim speed with density, as predicted by Eq. (19). This intriguing instability mechanism has been discussed by many groups theoretically (see, e.g., Ref. [8-16]), although an ultimate experimental validation is still pending.

A couple of notes are in order here. First, we should say that, although our derivation holds for run-and-tumble particle, it can be adapted without too much difference to the case of active Brownian particles (see also the minireview by T. Speck [17]), which change their direction of speed by rotational diffusion rather than through 
tumbling (you can try to adapt the derivation as an exercise!). Second, as mentioned at the beginning of this Section, the procedure to coarse grain a Boltzmann-like kinetic equation, based on a set of microscopic rules, to obtain a continuum model for macroscopic observables is a common one in nonequilibrium statistical physics, and in active matter physics. Often, though, one has to go beyond the equation for the density; e.g., for rod-like particles we would need to consider the polarisation or orientation field. The resulting maths can be much more complicated! To see some examples of a more complex gradient expansion in active systems, the reader can consult, e.g., Refs. $[18,19]$. We also note that steric effects will lead to local alignment of rod-like active particles: this alignment will promote local or global flocking - a discussion of generic aspects of the physics of flocking in active particles is given in the minireview by F. Ginelli [20].

Having discussed the basic physics of motility-induced phase separation, it is of interest to comment on the current state of research in the field. As anticipated, a lot of work has been done with systems only consisting of active self-propelled spheres [8-16]. However, only very recently has some work been carried out on activepassive mixtures, and this suggests that the phenomenology is even richer for such systems [21,22]. Similarly, while several papers discussed self-propelled rod-like particles, motility-induced phase separation for elongated particles remains less studied (see, though, the minireview [23] by Peruani in this issue). For instance, we know that a rod-like shape can lead to the formation of rotating clusters [25,26], but we do not yet know whether the scaling characterising cluster growth is the same as for the spherical case. Perhaps the most important issue to resolve, though, is the role of fluidmediated hydrodynamic interactions on motility-induced phase separation. Some papers suggest that hydrodynamics should suppress this phenomenon $[27,28]$, others that the ultimate answer depends on the details of particle-particle and particle-wall hydrodynamic interactions [29-31]. Again, most of the papers consider squirmers, which are spherical self-propelled particles; it would also of interest to know what happens for rods or elongated particles [32]. The role of fluid-mediated hydrodynamic interactions will also be discussed in the next Section, albeit in a slightly different context (that of active gels); several aspects of fluid dynamics associated with active systems are also discussed in the minireview [33] by R. G. Winkler.

\section{Including solvent-mediated interactions in hydrodynamic equations, and the generic instability of active gels}

In the previous Section, we studied the physics of a collection of self-propelled (runand-tumble) particles, neglecting the role of solvent-mediated interactions (this is because we only looked for an equation of motion for particle density, and not for the momentum of the active particles or an underlying fluid). Normally, active swimmers do so in a fluid, and the motion of a particle can affect, through this fluid, that of others. Furthermore, in order to move, active particles need to exert some force on the fluid (for example, in the case of bacteria, the movement is typically propelled by flagella which rotate on their back and set the nearby fluid into motion). This "stirring" leads to different consequences for collective behaviour with respect to motility itself, which we considered in the previous Section. Here we discuss how this microscopic stirring can be, once more, coarse grained to yield continuum equations of motion.

Let us consider a neutrally buoyant active particle: because there is no external force being exerted on it (as gravity does not contribute), we know that the total force exerted by the fluid on the particle (or vice versa) needs to sum up to zero. Therefore the simplest force distribution which an active particle can exert on its surrounding 
fluid is a force dipole, and this is also the starting assumptions of most theories of active fluids [34]. As a consequence, active "stirrers" are naturally associated with a direction, that of their force dipole. The forces then can either be directed "outwards" (from the centre of mass of the particles towards the fluid) or "inwards". In the first case the active particle is "extensile", or a pusher; in the second case it is called "contractile", or a puller $[34,35]$. Bacteria such as E. coli are pushers (their flagella push back the fluid), whereas Chlamydomonas, a well-studied alga (see the minireview by Polin [36]), swims by a different mechanism, and is effectively a puller [37].

We now want to provide a simple derivation (first presented in Ref. [35]), and also reported in [2]) of the hydrodynamic (coarse grained) equations of a suspension of dipolar stirrers: this system in active matter physics has been named an "active gel", or active fluid [38]. Again, we will be interested in a nonequilibrium phase transition that the "stirring" can lead to. It is remarkable that, as we will see shortly, the simplest theory applies both to polar particles, which move ("movers" in [2]) and to apolar particles, which stir the fluid without net movement ("shakers" in [2]).

To begin our derivation, we note that the force on the fluid can be written as the sum of the active forces exerted by all the $N$ stirrers, as follows,

$$
\sum_{j=1}^{N} \sum_{i=1}^{2} \mathbf{F}_{j, i}=\mathbf{F}_{\text {active }}
$$

In the Equation above $\mathbf{F}$ denotes the force, the subscript $j$ denotes particle number and $i$ goes from 1 to 2 as every particle exerts two forces being an active dipole. Let us now consider a swimmer of length $b+b^{\prime}$; which we imagine as a one dimensional infinitesimally thin rod - the direction of the $j$-th $\operatorname{rod}, \mathbf{P}_{j}$, is that of the force dipole of the stirrer; this need not coincide with the direction of motion, as the particle could be a shaker. One end of the swimmer is $b$ away from the center of mass $\mathbf{r}_{i}$ of the rod, the other end $b^{\prime}$. Hence the force distribution is

$$
\mathbf{F}_{\text {active }}=\sum_{j=1}^{N}\left[\mathbf{f}_{j} \delta\left(\mathbf{r}-\mathbf{r}_{j}-b \mathbf{P}_{j}\right)-\mathbf{f}_{j} \delta\left(\mathbf{r}-\mathbf{r}_{j}+b^{\prime} \mathbf{P}_{j}\right)\right] .
$$

In the limit of $b, b^{\prime} \rightarrow 0$ (point-like dipoles), we get

$$
F_{\alpha}^{\text {active }}=\sum_{j=1}^{N} f_{j, \alpha}\left[-b P_{j \beta} \partial_{\beta} \delta\left(\mathbf{r}-\mathbf{r}_{j}\right)-b^{\prime} P_{j \beta} \partial_{\beta} \delta\left(\mathbf{r}-\mathbf{r}_{j}\right)\right]
$$

Now, as $f_{j \alpha}=f P_{j \alpha}$, the previous equation becomes:

$$
F_{\alpha}^{\text {active }}=-f\left(b+b^{\prime}\right) \partial_{\beta} \sum_{j=1}^{N} P_{j \alpha} P_{j \beta} \delta\left(\mathbf{r}-\mathbf{r}_{j}\right) .
$$

We now again wish to do a coarse graining. This time, we will use a more handwaving approach than in last Section. For large $N$, we define the coarse grained density $\rho$ (particle density) and the coarse grained tensor $Q_{\alpha \beta}$ (this is the nematic tensor, measuring the orientational order of the active dipoles) as follows [2]

$$
\sum_{j=1}^{N} \delta\left(\mathbf{r}-\mathbf{r}_{j}\right)=\rho(\mathbf{r})
$$



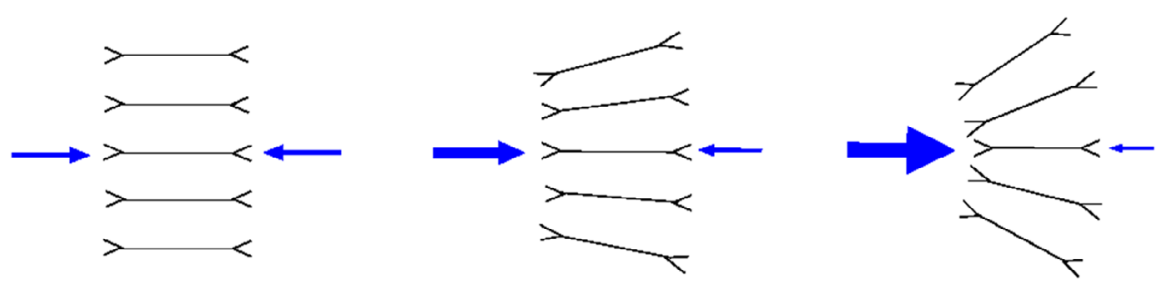

Fig. 2. Sketch of how the "generic instability" works in the active ordered phase, for a contractile active fluid (i.e., for inward active dipoles). When there is no distortion, the active forces, shows as blue arrows, balance (left). A splay leads to an imbalance which creates a flow to the right (middle); this creates further splay (right), hence an instability. The final pattern depends on stabilising terms such as viscosity and elastic constants. This argument was first proposed in Ref. [35].

$$
\sum_{j=1}^{N} \delta\left(\mathbf{r}-\mathbf{r}_{j}\right) P_{j \alpha} P_{j \beta}=\rho(\mathbf{r}) Q_{\alpha \beta}(\mathbf{r}) .
$$

Equation (23) thus can be written as:

$$
F_{\alpha}^{\text {active }}=-\zeta \partial_{\beta}\left(\rho Q_{\alpha \beta}\right),
$$

where $\zeta=f\left(b+b^{\prime}\right)$ is the activity of the swimmers. In Eq. (26) we can write the force as the divergence of an active stress tensor, $\partial_{\beta} \Pi_{\alpha \beta}^{\text {active }}$, where

$$
\Pi_{\alpha \beta}^{\text {active }}=-\zeta \rho Q_{\alpha \beta},
$$

Looking back at our derivation, we can see that for positive $\zeta$, the systems corresponds to a collection of extensile particles (e.g., bacteria); whereas $\zeta<0$ is the case of contractile rods (such as Chlamydomonas, another example is a collection of a network of actin fibres and myosin motors, which exert contractile forces on the actin fibres). The hydrodynamics is significantly different in the two cases [2,39-44]. Another important fact to observe is that, as anticipated, this first order contribution to the hydrodynamic stress tensor of the active fluid, Eq. (27) is the same for movers and shakers, as $\zeta$ is proportional to $b+b^{\prime}$, hence does not vanish when $b=b^{\prime}$ for a shaker.

The active stress in Eq. (27) has important effects on the statistical physics of suspensions of swimmers (or stirrers). One of these is that this forcing, when large enough, triggers a nonequilibrium transition between a quiescent phase, where the coarse grained velocity field of the solvent (or of the active particles) is zero (so while particles may swim individually there is no collective velocity, or swarming, of them as a whole), to a phase where there is spontaneous flow, which is sustained by the internal dipolar force distribution.

There have been many studies of this transition, by means of linear stability analysis $[2,35,45,46]$ or numerical simulations of the hydrodynamic equations of motion [47-53]. Here we simply wish to understand the mechanism of this transition, in one particular case where dipoles are initially orientationally ordered (e.g., because each active particle is rodlike and the concentration is sufficiently large to trigger a transition to the nematic phase [54]). Let us consider the situation sketched in Fig. 2, and to fix the ideas let us focus on the case of contractile dipoles. When the nematic order is perfect (left panel), the force dipoles balance and there can be no flow. However, imagine introducing a small splay deformation (middle panel): now the density 
(A)
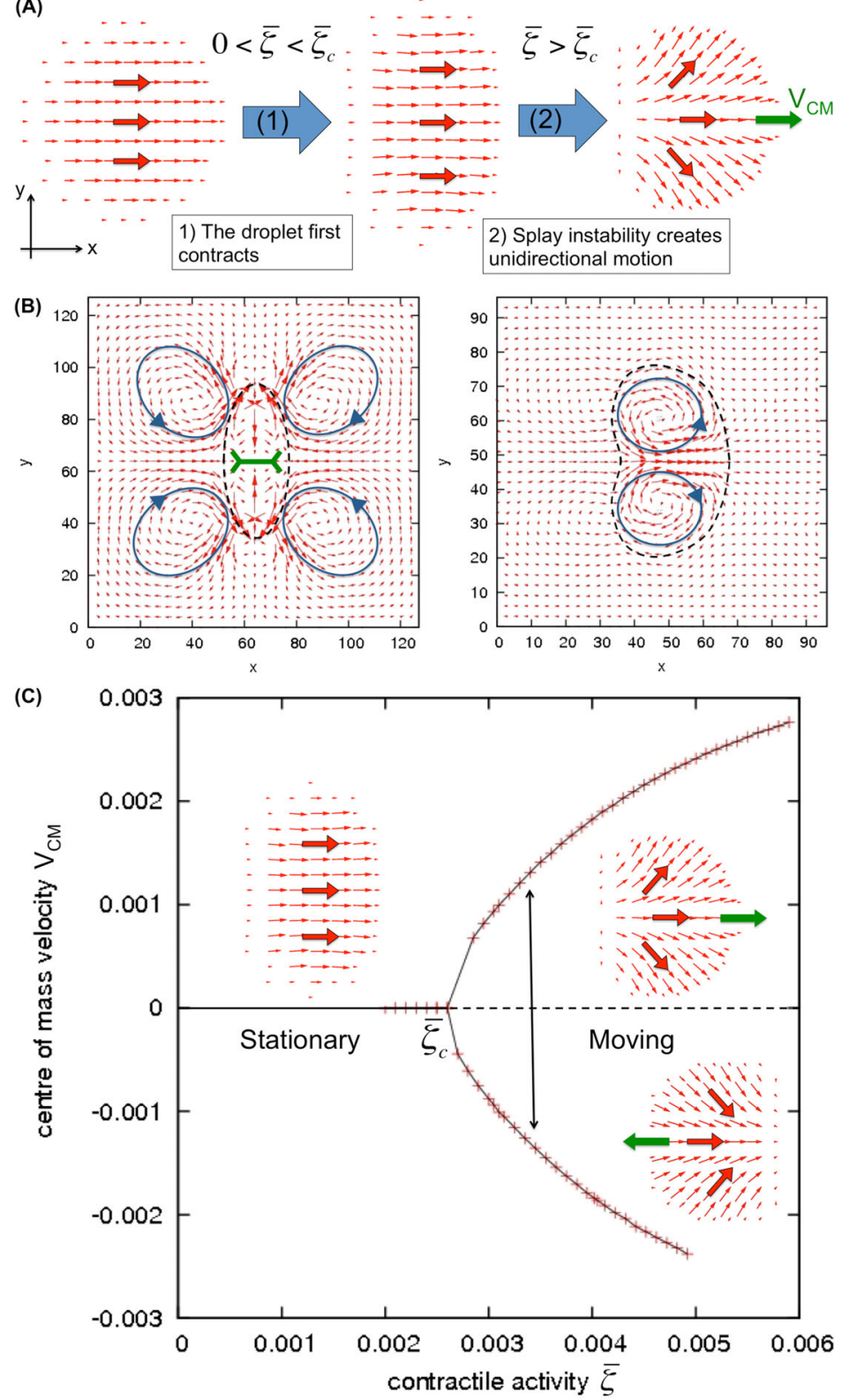

Fig. 3. The generic instability can set contractile active gel droplets into motion. Here, a contractile active droplet is created through a free energy density which favours the formation of an active polar nematic droplet within an isotropic background, see Ref. [55] for details. (A) Steady state configurations of contractile active droplet. The (red) arrows show the polarisation field $\mathbf{P}(\mathbf{r}, t)$. Upon increasing the contractile activity, here denoted as $\bar{\zeta}$, the droplet elongates perpendicular to the direction of its polarisation, and then becomes unstable with respect to splay deformations at a critical activity $\bar{\zeta}_{c}$. The mechanism is the "generic instability" which is reviewed in Fig. 2. When the droplet splays, it also spontaneously moves in the direction of the green arrow, due to the imbalance of active forces. (B) The left plot shows the velocity field of the droplet at $\bar{\zeta}<\bar{\zeta}_{c}$ which is quadrupolar. The right plot shows the velocity field in the self-motile regime. The boundary of the droplet is given by the dashed line. (C) Bifurcation diagram showing spontaneous symmetry breaking from a uniform and stationary state to a splayed and moving state as the contractile activity is increased. Figure taken from Ref. [55], with permission. (This figure is subject to copyright protection and is not covered by a Creative Commons license.) 
of contractile forces to the left of the splayed pattern is larger than on the right, as a result a flow sets up: because of the rodlike nature of the active dipoles, this flow causes further splay, hence the system is unstable and starts to flow macroscopically. If we invert the sign of the force dipoles, and by repeating this argument you should convince yourself that an extensile fluid is instead stable with respect to splay (try this as an exercise). However, this does not mean that the extensile gel is unconditionally stable, as a bend fluctuation would instead grow (again this can be understood with an argument similar to that sketched in Fig. 3).

These arguments showing that contractile and extensile gels are unstable to splay and bend respectively were first discussed in Refs. [2,35]. These are very general, and can have remarkable consequences. For instance this so-called "generic instability" can set into motion a droplet of active gel within an isotropic fluid [55-57]. This interesting phenomenon is depicted in Fig. 3: within an active contractile droplet, a splay fluctuation creates an imbalance of active forces which simultaneously sustains the elastic deformations and propels the droplet (to the right in the schematics in Fig. 3, panel (A)). The self-motile droplet has an internal flow field which consists of two opposing vortices (Fig. 3, panel (B)). The nonequilibrium transition between quiescent and self-motile droplet can be seen to be continuous, for instance by plotting the centre of mass velocity as a function of contractile activity (Fig. 3, panel (C)). This contractility-driven (or, in general, activity-driven) motility might possibly provide a framework to understand the motility of cells, or cell extracts, in 3D $[58,59]$. Simulations have also been performed where contractility is combined with treadmilling - another active process which consists in the polymerisation of actin fibres, which leads to motility on a substrate. These simulations give rise to a variety of cell morphologies, some of these resemble quite closely motile cells [60]. Other recent theoretical work on active gel droplets can be found, among others, in Refs. [61-65]. It is clear that a lot of work remains to be done in this area, both in providing a theoretical framework to understand the simulation results, and in taking forward the simulation models to provide more and more realistic descriptions of cell extracts and motile cells.

\section{Conclusion}

In summary, we have seen in this minireview two examples of coarse graining procedures to go from a set of microscopic rules to continuum models which track the evolution of macroscopic fields such as density, momentum, etc. This procedure is widely used in nonequilibrium statistical physics, and therefore it is useful in the physics of active matter and self-propelled particles as well. While the method can be applied in general (although the calculations can quickly become cumbersome in practice), we have also shown two specific active matter examples where the macroscopic (hydrodynamic) equations describe nonequilibrium transitions in these systems. For both examples, we have also briefly sketched open questions and some aspects which are the topic of active research.

For the interested reader, good and comprehensive review articles covering the material discussed in this minireview (and a lot more) are [4,34,66-68]. Within this issue, there are several minireviews which are directly relevant to, and complementary to, the topics we have considered here. In particular, the physics of the Vicsek model is discussed in [20], some complementary features of motility-induced phase separation for active Brownian particles are covered in [17], several aspects of the importance of fluid-mediated hydrodynamics in active systems are dealt with in [33], whereas active Brownian rods are the topic of the minireview in [23]. 


\section{References}

1. G. De Magistris, D. Marenduzzo, Physica A 418, 65 (2015)

2. Y. Hatwalne, et al., Phys. Rev. Lett. 92, 118101 (2004)

3. W.C.K. Poon, Proceedings of the International School of Physics "Enrico Fermi", Course CLXXXIV "Physics of Complex Colloids", edited by C. Bechinger, F. Sciortino, P. Ziherl (IOS, Amsterdam: SIF, Bologna, 2013), p. 317

4. M.E. Cates, Rep. Progr. Phys. 75, 042601 (2012)

5. K. Huang, Statistical Mechanics (Wiley \& Sons, USA, 1986)

6. S. Chapman, T.G. Cowling, The Mathematical Theory of Non-uniform Gases: an Account of the Kinetic Theory of Viscosity, Thermal Conduction, and Diffusion in Gases (Cambridge University Press, 1990)

7. M.E. Cates, D. Marenduzzo, I. Pagonabarraga, J. Tailleur, Proc. Natl. Acad. Sci. USA 107, $11715(2010)$

8. Y. Fily, M.C. Marchetti, Phys. Rev. Lett. 108, 235702 (2012)

9. S. Henkes, Y. Fily, M.C. Marchetti, Phys. Rev. E 84, 040301 (2011)

10. Y. Fily, S. Henkes, M.C. Marchetti, Soft Matter 10, 2132 (2014)

11. G.S. Redner, M.F. Hagan, A. Baskaran, Phys. Rev. Lett. 110, 055701 (2013)

12. G.S. Redner, A. Baskaran, M.F. Hagan, Phys. Rev. E 88, 012305 (2013)

13. S.R. McCandlish, A. Baskaran, M.F. Hagan, Soft Matter 8, 2527 (2012)

14. I. Buttinoni et al., Phys. Rev. Lett. 110, 238301 (2013)

15. A. Wysocki, R.G. Winkler, G. Gompper, EPL 105, 48004 (2014)

16. J. Stenhammar, et al., Phys. Rev. Lett. 111, 145702 (2013)

17. T. Speck, Eur. Phys. J. Special Topics 225, 2287 (2016)

18. E. Bertin, M. Droz, G. Gregoire, J. Phys. A 42, 445001 (2009)

19. F.D.C. Farrell et al., Phys. Rev. Lett. 108, 248101 (2012)

20. F. Ginelli, Eur. Phys. J. Special Topics 225, 2099 (2016)

21. J. Stenhammar, R. Wittkowski, D. Marenduzzo, M.E. Cates, Phys. Rev. Lett. 114, $018301(2015)$

22. A.Y. Grosberg, J.F. Joanny, Phys. Rev. E 92, 032118 (2015)

23. F. Peruani, Eur. Phys. J. Special Topics 225, 3001 (2016)

24. A.P. Solon, J. Stenhammar, R. Wittkowski, M. Kardar, Y. Kafri, M.E. Cates, J. Tailleur, Phys. Rev. Lett. 19, 198301 (2015)

25. A. Suma, G. Gonnella, D. Marenduzzo, E. Orlandini, EPL 108, 56004 (2014)

26. J. Schwarz-Linek, C. Valeriani, A. Cacciuto, M.E. Cates, D. Marenduzzo, A.N. Morozov, W.C.K. Poon, Proc. Natl. Acad. Sci. USA 109, 4052 (2012)

27. R. Matas-Navarro, R. Golestanian, T.B. Liverpool, S.M. Fielding, Phys. Rev. E 90, $032304(2014)$

28. R. Matas-Navarro, S.M. Fielding, Soft Matter 11, 75254 (2015)

29. F. Alarcon, I. Pagonabarraga, J. Mol. Liq. 185, 56 (2013)

30. A. Zoettl, H. Stark, Phys. Rev. Lett. 112, 118101 (2014)

31. A. Furukawa, D. Marenduzzo, M.E. Cates, Phys. Rev. E 90, 022303 (2014)

32. J. De Graaf, H. Menke, A.J.T.M. Mathijssen, M. Fabritius, C. Holm, T.N. Shendruk [arXiv:1602.07560]

33. R.G. Winkler, Eur. Phys. J. Special Topics 225, 2079 (2016)

34. S. Ramaswamy, Ann. Rev. Cond. Matt. 1, 323 (2010)

35. R.A. Simha, S. Ramaswamy, Phys. Rev. Lett. 89, 058101 (2002)

36. R. Jeanneret, M. Contino, M. Polin, Eur. Phys. J. Special Topics 225, 2141 (2016)

37. M. Polin, et al., Science 325, 487 (2009)

38. K. Kruse, et al., Phys. Rev. Lett. 92, 078101 (2004)

39. M.E. Cates, et al., Phys. Rev. Lett. 101, 068102 (2008)

40. D. Marenduzzo, E. Orlandini, J.M. Yeomans, Phys. Rev. Lett. 98, 118102 (2007)

41. S.M. Fielding, D. Marenduzzo, M.E. Cates, Phys. Rev. E 83, 041910 (2011)

42. L. Giomi, T.B. Liverpool, M.C. Marchetti, Phys. Rev. E 81, 051908 (2010)

43. G. Foffano, et al., Phys. Rev. Lett. 109, 028103 (2012)

44. G. Foffano, et al., Eur. Phys.J. E 35, 98 (2012) 
45. R. Voituriez, J.F. Joanny, J. Prost, Europhys. Lett. 70, 404 (2005)

46. R. Voituriez, J.F. Joanny, J. Prost, Phys. Rev. Lett. 96, 028102 (2006)

47. D. Marenduzzo, et al., Phys. Rev. E 76, 031921 (2007)

48. D. Saintillan, M.J. Shelley, Phys. Rev. Lett. 100, 178103 (2008)

49. D. Saintillan, M.J. Shelley, Phys. Fluids 20, 123304 (2008)

50. E. Barath, M.J. Shelley, D. Saintillan, Phys. Fluids 25, 070607 (2013)

51. L. Giomi, L. Mahadevan, B. Chakraborty, M.F. Hagan, Phys. Rev. Lett. 106, 218101 (2011)

52. L. Giomi, M.J. Bowick, X. Ma, M.C. Marchetti, Phys. Rev. Lett. 110, 228101 (2013)

53. E. Tjhung, M.E. Cates, D. Marenduzzo, Soft Matter 7, 7453 (2011)

54. R.A.L. Jones, Soft Condensed Matter (Oxford University Press, New York, 2002)

55. E. Tjhung, D. Marenduzzo, M.E. Cates, Proc. Natl. Acad. Sci. USA 109, 12381 (2012)

56. C.A. Whitfield, et al., Eur. Phys. J. E 37, 8 (2014)

57. L. Giomi, A. de Simone, Phys. Rev. Lett. 112, 147802 (2014)

58. R. Poincloux, O. Collin, F. Lizarraga, M. Romao, M. Delbray, M. Piel, P. Chavrier, Proc. Natl. Acad. Sci. USA 108, 1943 (2011)

59. R.J. Hawkins, R. Poincloux, O. Benichou, M. Piel, R. Voituriez, Biophys. J. 101, 1041 (2011)

60. E. Tjhung, A. Tiribocchi, D. Marenduzzo, M.E. Cates, Nat. Comm. 6, 5420 (2015)

61. D. Khoromskaia, G.P. Alexander, Phys. Rev. E 92, 062311 (2015)

62. F. Ziebert, S. Swaminathan, I.S. Aranson, J. Roy. Soc. Interf. 9, 1084 (2012)

63. J. Loeber, F. Ziebert, I.S. Aranson, Soft Matter 10, 1365 (2014)

64. M. Schmitt, H. Stark, EPL 101, 44008 (2013)

65. P. Recho, T. Putelat, L. Truskinovski, Phys. Rev. Lett. 111, 108102 (2013)

66. M.E. Cates, J. Tailleur, Ann. Rev. Cond. Matt. Phys. 6, 219 (2015)

67. M.C. Marchetti, et al., Rev. Mod. Phys. 85, 1143 (2013)

68. J. Elgeti, R.G. Winkler, G. Gompper, Rep. Prog. Phys. 78, 056601 (2015)

Open Access This is an Open Access article distributed under the terms of the Creative Commons Attribution License (http://creativecommons.org/licenses/by/4.0), which permits unrestricted use, distribution, and reproduction in any medium, provided the original work is properly cited. 